\title{
Monitoring wheel defects on a metro line: system description, analysis and results
}

\author{
M. Seco, E. Sanchez \& J. Vinolas \\ CEIT and Tecnun (University of Navarra), Spain
}

\begin{abstract}
This paper presents a system developed to monitor wheel defects on metro vehicles in order to reduce annoyance and infrastructural damage due to noise and vibrations. Information about the wheel state and its evolution can be added to the mere mileage to improve the efficiency of the reprofiling decisions. In addition, sudden defects, that could potentially be severe, are detected and an alarm is raised to take the necessary actions.

Some other systems have been developed to monitor wheel state by means of measuring the vibrations or noises generated by vehicles; however, the case of a metro is somewhat distinct. Wheel thread defects on a metro vehicle are not necessarily related to overloads or rolling contact fatigue. Damage to infrastructure and noise emission generated by small defects are particularly important due to the high frequency of the service and the proximity of the track to residential buildings, especially in the case of an open-air section that runs though a neighbourhood as some sections of the Metrobilbao.

The system measures vibrations at a specific location on the rail as the vehicle passes over it, then analyzes these signals and stores the information in a specific database that can be easily queried from any computer on the intranet. The system has been installed on the Metrobilbao line and tested for three years. During this period, more than 8500 trains, or 272000 wheels, have passed over the accelerometers and corresponding data was stored in a database. 448 wheels threads were physically measured in order to check the accuracy of the system. This data has been used to fit the statistics for new or newly machined wheels and estimate the alarm threshold, and finally tuned in agreement with the maintenance workshop.
\end{abstract}

Keywords: wheelflat, monitoring, wheel/rail contact. 


\section{Introduction}

The Railway industry is under increasing pressure due to the economic competition and requirements on comfort, on noise emission and safety for passenger and goods.

Simultaneously, a gain in speed and axle load results in increasing stresses in the wheel/rail contact, raising wear and maintenance costs. Little wear or small defects on rolling surfaces may result in overloading the wheels, bearings, rails and sleepers together with greater vibrations and acoustic emission. The most commonly reported wheel defects are known as flats and shells and it is estimated that $\$ 90$ million dollars are spent in the USA replacing 125000 defective wheels a year [1].

All of these problems are the reason for the growing interest in the Railway industry in preventive and predictive maintenance of infrastructures and rolling stock that has led to the development of different systems to detect wheel defects. Since the first "Wheel Impact Load Detector" (WILD) device was installed in the USA in 1983 [2], different systems and technologies have been proposed or developed [3-19].However, correct quantification and monitoring are not definitely solved problems.

The generalized method to detect faulty wheels is based on the measurement of rail loads or vibrations, for wheel defects generate great impact loads, vibrations and high stresses on the rail. Different techniques have been used to measure the vibrations: accelerometers on the rail, strain gauges, load cells, etc. and a variety of algorithms have been developed to analyze measured signals.

Systems based on accelerometers are the Wheel Monitoring System (WMS) [8], the Wheel Impact Monitor (WIM) [10] and the one presented by Skarlatos et al. [18]. Other methods, [12, 13, 17], use the cepstum function [20] to analyse the signals from piezoelectric cables and accelerometers.

The Wheel Condition Monitor (WCM) [19], and the TrackAlert® system [5] use both accelerometers and strain sensors installed on the rail while other systems use load cells or other equivalent devices to measure load peaks instead of accelerations $[4,6,11]$.

Feng et al. [15] have developed a different device, based on the measurement of the vertical movement of the wheel flange by means of a rule attached to the rail. The principal limitation of the system is the relative low train speed required to work properly and its mechanical complexity.

Jodon, inc [11] propose a system based on microwave that measures the wheel profile and would be also capable of measuring wheel defects over the rolling band.

This article presents a low-cost monitoring system, based on accelerometers, developed by CEIT and Metrobilbao that was placed and successfully tested in the Metrobilbao railway system. Principal advantages of the system are precisely its low cost and simplicity.

This paper includes a system description on section 2 , the signal processing and treatment on section 3 , the experimental verification and alarm threshold on section 4 and conclusions on section 5 . 


\section{System description}

When an impact is applied to the rail, vibrations are propagated along the track decaying with distance. It is then necessary to know both the decay rate and the distance from the impact point to estimate the accuracy of the measurements.

Although extensive studies of the wave propagation along the rail have been published [21, 22], measured data have been used to simply estimate the specific decay rate of the registered vibrations as the flat impacts repetitively along the rail. An example of the registered signal corresponding to a Metrobilbao vehicle with a wheelflat is shown in Figure 1:.Y-axis shows the RMS values of the acceleration in $\mathrm{dB}$ during the train passing by (X-axis). Impacts on the rail are due to a defect that affects the rolling contact point at every turn of the wheel.

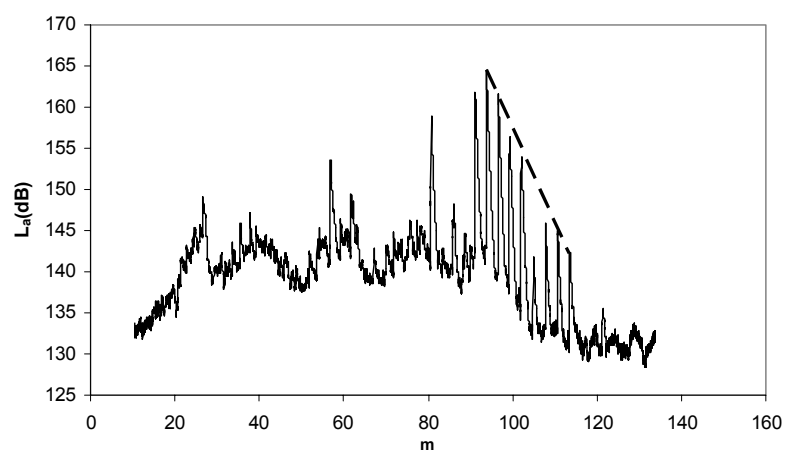

Figure 1: $\mathrm{La}\left(\mathrm{ref}=1 \mu \mathrm{m} / \mathrm{s}^{2}\right)$ of the acceleration signal for a Metrobilbao vehicle with a wheelflat in the rear last wheel. The slope of the fitted line is the spatial track decay rate for the impacts due to wheel defects.

A regression line can be fitted to the maximum of the different impact echoes shown in Figure 1: and the slope of the fitted line is thus the spatial decay of the excitation.

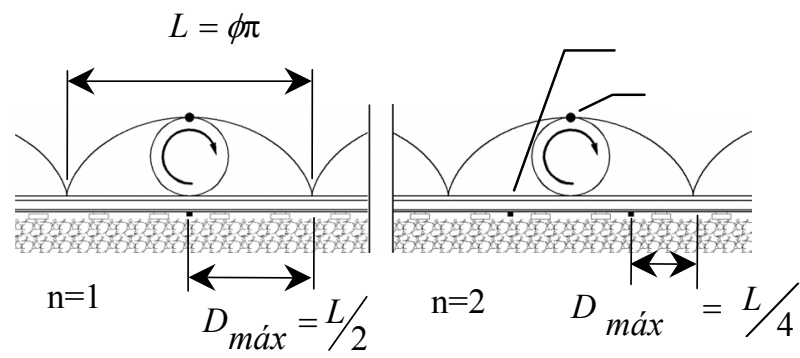

Figure 2: Defect paths and Dmax for 1 and 2 sensors on rail.

As the decay rate is rail dependent, the only way to achieve more precise measurements of the vibrations is to minimize the maximum distance $\left(D_{\max }\right)$ in which the excitation may occur because the bigger is the distance between the 
impact and the measurement point, the bigger is the underestimation of the signal. The way to minimize $\mathrm{D}_{\max }$ is to increase the number of sensors installed on the rail $[10,19]$. For a single sensor scheme $D_{\max }$ is half the circumference length, with 2 sensors it is a quarter of the wheel circumference, etc (Table 1: ).

Finally, 2 accelerometers have been installed at each measuring point looking for a half-way between costs and maximum admissible error.

Table 1: Different options for the accelerometers scheme.

\begin{tabular}{|c|c|c|c|c|c|}
\hline \multirow[t]{2}{*}{$\mathrm{n}$} & \multirow[t]{2}{*}{ Mounting Scheme } & $x \longleftrightarrow$ & Dmax & \multicolumn{2}{|c|}{ Attenuation } \\
\hline & & $\mathrm{m}$ & $\mathrm{m}$ & $\Delta \mathrm{La}(\mathrm{dB})$ & $\mathrm{a} / \operatorname{amax}(\%)$ \\
\hline 1 & பட பனேபட & -- & $\mathrm{L} / 2=1.32$ & -1.584 & $83.3 \%$ \\
\hline 2 & படனேபனப & 1.32 & $\mathrm{~L} / 4=0.66$ & -0.79 & $91.3 \%$ \\
\hline 3 & பட பण口யப & 0.88 & $\mathrm{~L} / 6=0.44$ & -0.53 & $94.1 \%$ \\
\hline 4 & पனுणூனு & 0.66 & $\mathrm{~L} / 8=0.33$ & -0.4 & $95.5 \%$ \\
\hline
\end{tabular}

X: Distance between accelerometers. Where $x=\frac{\phi \pi}{n}$ with $\mathrm{n}=$ number of accelerometers and $\varnothing=$ wheel diameter.

Dmax: Maximum disance between the measurement point and the impact point $\Delta$ La: Theoretical decay in $\mathrm{dB}$, where $\Delta L_{a}=D R \times D_{\max }$ and $\mathrm{DR}$ is the estimated Decay Rate.

a/amax: Maximum underestimation of the real excitation due to spatial decay.

The main components of the developed device are the accelerometer matrix under the rail, a wayside sonometer, signal transmission circuits, and the computer that controls the system, analyzes the signals and stores data. A couple of proximity detectors activate the system when a train approaches and are also used to estimate the speed of the train.

Figure 3: shows and schematic of the device installed close to a station in the Metrobilbao network. Notice that the equipments are spread into three zones:

a. Control booth: where the computer and the acquisition electronics are placed.

b. Bend zone: 4 accelerometers, a sonometer and a proximity detector.

c. Straight zone: 4 accelerometers, and a proximity detector.

Some of the accelerometers have been installed on a bend because during turning the wheel-rail contact point moves along the wheel and rail profiles and this may allow one to detect different out of the rolling band defects.

A1 to A4 accelerometers on Figure 3: are far over 200m from the computer so a current transmitter circuits system has been installed to avoid noise and signal attenuation. Designed voltage-to-current converters transmit conditioners signals and current-to-voltage converters reverts the signals to the acquisition card inputs. 


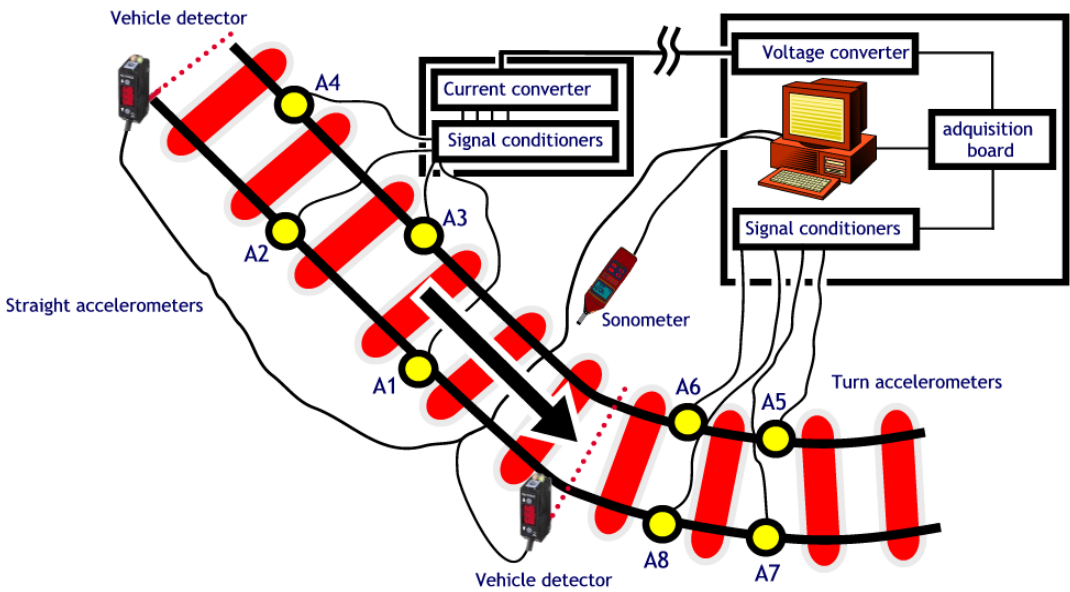

Figure 3: The system has been installed in the Metrobilbao network.
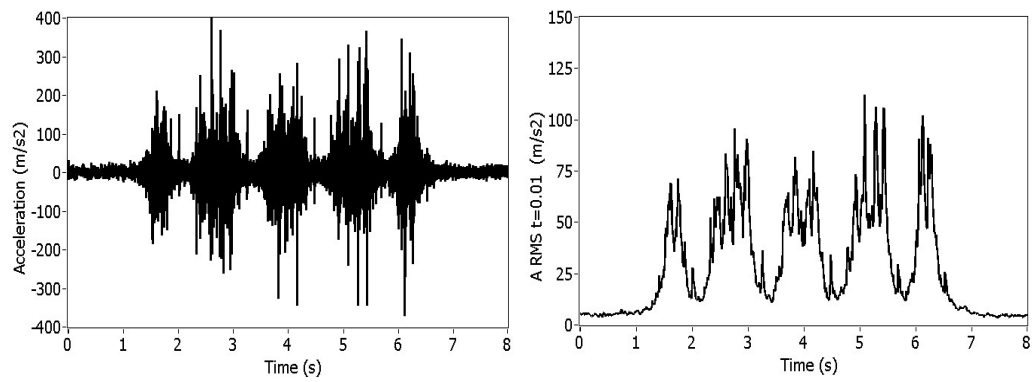

Figure 4: Rail vibrations during the train passing. Train without defective wheels. Left) original signal. Right) RMS integrated signal.

The sampling rate chosen for the signal acquisition is $2 \mathrm{kHz}$, since it was verified that the acceleration spectra of interest is below $1 \mathrm{kHz}$ [17]. A sample for the registered rail acceleration pattern for a defect-free train is shown in Figure 4:. Obviously, the higher is the sampling rate, the better the signal but $2 \mathrm{kHz}$ is high enough for monitoring necessities.

Accelerometers are fastened with a magnet and it has been used for it is simple, easy to install and move and it has remained successfully fixed during the system development.

\section{Signal analysis and software}

Acquired data is analyzed and stored and that makes post-processing possible because none of the data is deleted or modified during analysis allowing the to 
alarm thresholds to be updated or to study particular trends, peculiarities or outstanding values without waiting for new data.

Some parameters, apart from the train identification, needed to carry the monitoring out, are the train speed and the train geometry.

The variable used for monitoring is the root mean square of the signal (Figure 4:).

Exhaustive tests have been carried out looking for the optimum value for the time step and finally it has been chosen in order to assure that faulty and non-faulty levels are clearly different and variability within the same state is minimal (Figure 5:).

The integrated values from each of the wheels for every vehicle transit are stored in a database. This database is the core of the system and different data requests are used for report warnings, defect evolution or instant wheels state. This configuration allows great flexibility in order to carry out different studies.

The system makes use of a couple of sensors within each of the rails to minimize the variability of the measures. Data from both sensors, corresponding to each of the wheels, are analysed but only the maximum values from both of the signals are stored on the database as corresponding to the level of each of the wheels.

The system raises the alarm when a defective wheel is detected but available working time and, especially, economical and wheel durability are, up to now, the leading parameters on the turning or changing wheels decisions. For this reason, the alarm threshold must include not only the mere defect importance but also the repeatedly machining costs in order to optimize the wheel life-cycle

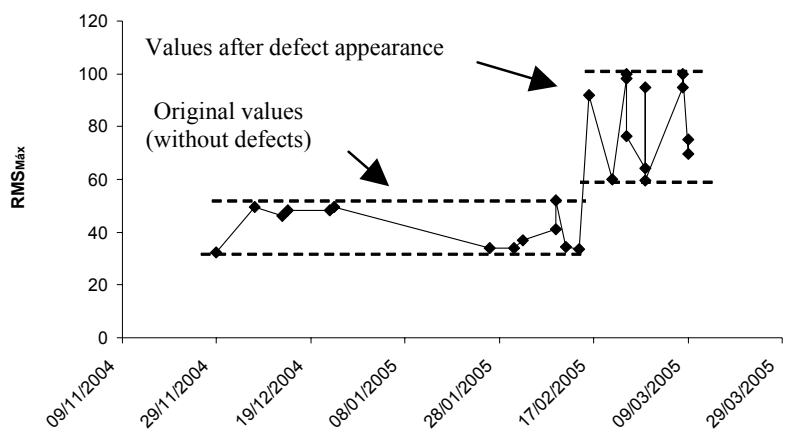

Figure 5: Evolution of the $\mathrm{RMS}_{\Delta t=0.04}$ values with the apparition of a defect.

\section{Alarm threshold tuning and experimental verification}

Some studies have been published about the allowable defect magnitude in order to avoid rail damage. However, the problem in metro systems is more related to generated noise than to rail damage. In fact, great defects, when they occur, are currently quickly detected while a relative high noise level is particular annoying in open air tracks that are close to buildings and houses, even more with high 
train frequency and if the service begins soon in the morning a finishes late at night.

The first approach to the alarm level tuning is to discern between 'perfect' and defective wheels. To do so, logarithms of the $\mathrm{RMS} \Delta \mathrm{t}=0,04$ values corresponding to new or just turned wheels are used to fit a reduced extreme values distribution as seen in next figure.
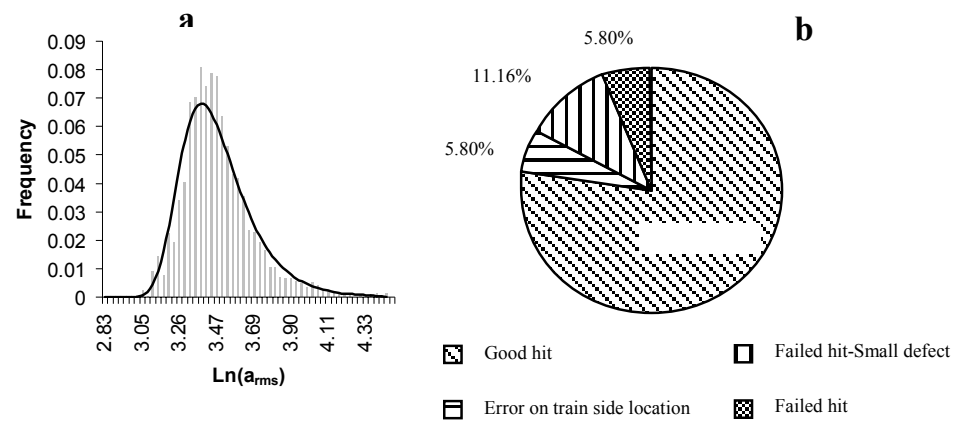

Figure 6: a) Experimental histogram of the logarithm of the acceleration for perfect or newly machined wheels $(n=2847)$. Black line corresponds to the theoretical reduced extreme value distribution adjusted to the experimental values. b) Reliability of the device during machining process supervision $(n=448)$.

Fitting is performed by means of maximum likelihood estimators and $99 \%$ confidence intervals are proposed to identify out of limit values that are considered to correspond with faulty wheels.

In order to involve maintenance department and workshop operators in the system developing, weekly alarms were reported as well as information about the state of the vehicle scheduled to be turned. This has allowed making use of the workshop experience to conform alarm thresholds to operative values.

Tests included inspection of the turning of 448 wheels and $77 \%$ resulted in good agreement with the device estimation while $5.8 \%$ were considered to have failed. The rest of the failed estimations may be explained as follows:

A) The defect has been localized in the same axle but in the opposite wheel. Such mistake may be due to practical error of the workshop operator when reporting his observations.

B) Alarms with level 1 out of 4, which are not reported as having a defect may be caused because the device raises a low level alarm due to effects not related to wheel defects or it may be due to visual misdetection of a small defect during machining operations. Any way, these faults were not critical because the alarm level was low and it could be solved raising the alarm threshold level.

Figure 7: shows an example of the correlation between the monitoring based alarms and the defects found during the turning operation. 


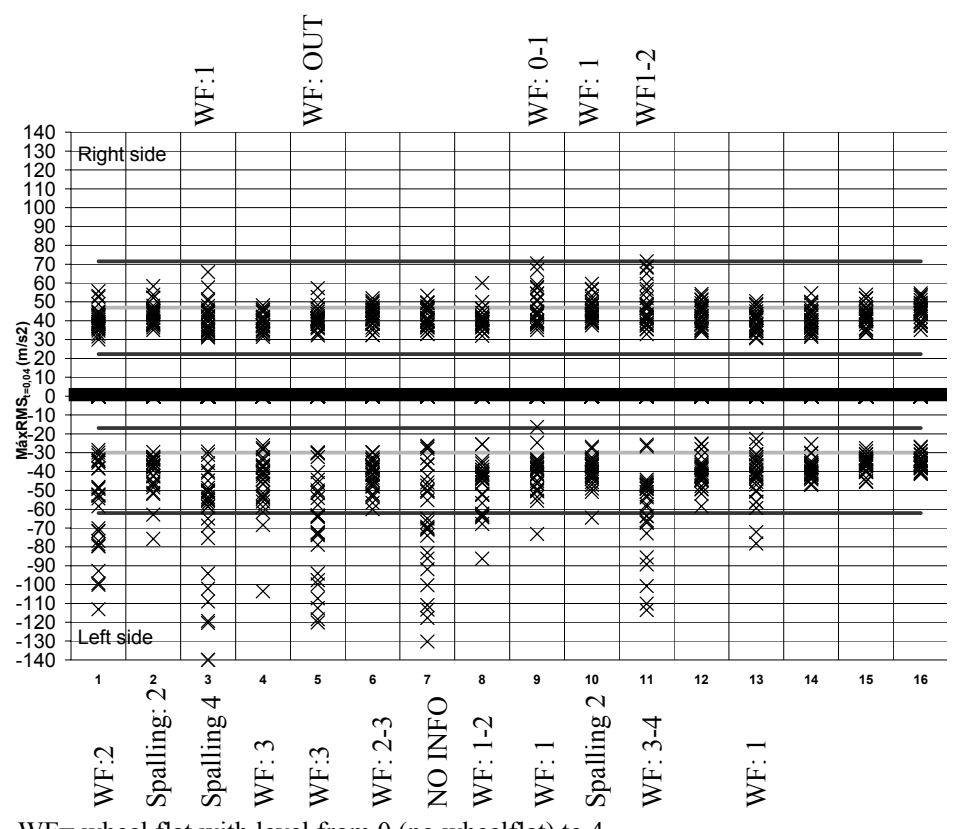

$\mathrm{WF}=$ wheel flat with level from 0 (no wheelflat) to 4

On the $5^{\mathrm{TH}}$ wheel on the right a small flat was reported to be out of the rolling band.

Figure 7: Comparison between data from the monitoring system (plot) and defect found during wheel re-profiling process (up and down).

The central graph shows vehicle monitoring information. The axis of abscissas shows the different wheel number of the monitored vehicle and the axis of ordinates measures the maximum acceleration values. The graph includes data from the right side wheels (up) and the left ones (bottom) and summarizes ten passages of the studied train. Horizontal lines delimit theoretic normal values and outstanding data are considered to correspond to faulty wheels. The figure also shows information reported from machining workshop, it includes results from visual inspection, machining incidents and other information.

\section{Conclusions}

Defects on the rolling band in train wheels are the cause of large impacts on the rail that generate noise and vibrations. With this work it has been proved that measuring these rail vibrations is a reliable method to determine the presence and magnitude of wheel defects. This paper presented a detailed study of the existing and currently developing technology in measuring techniques and analyzing algorithms. Also, it has been proved to be precise enough to use two accelerometers beneath each rail. Additionally, it is interesting to install accelerometers in different positions, straight line and turning zone because defects in different locations on the wheel surface are so detected. 
Speed estimation is performed by means of the presence detector signals and adequate accuracy has been achieved. Speed accuracy is important in order to discard that measures corresponding to vehicles running with out of normal speed. In addition, vehicle speed is used during the signal analysis to correlate the signal section with its corresponding wheel.

In this study 448 wheels have been studied, comparing the predicted status with data from the machining process. Only $5,8 \%$ of the predicted status clearly failed while the other $5,8 \%$ of the predictions resulted in minimal defect misdetection.

The fully automatic operation of the system is under development by means of the automatic identification of the vehicle and the connection of the system with the data network of Metrobilbao.

\section{Acknowledgements}

Developing the system would have been an impossible task without the support of Metrobilbao. They have always trusted that the system would finally work so they have financed the work and have been at our beck and call to use their installations and rolling stock to carry out measurements, many times at night.

\section{References}

[1] R.V. Dukkipati and R. Dong, Impact loads due to wheel flats and shells, Vehicle System Dynamics, 31(1),pp. 1-22, 1999

[2] Federal Railroad Administration (U.S. Department of Transportation), Dynamic Wheel Load Detector Extends Life of Concrete Railroad Ties, TR news, January-February, 1987

$\begin{array}{lll}\text { AEA Technology: } \quad \text { Wheelchex } & \\ \text { http://www.aeat.co.uk/rail/what/products/wheelchex.htm }\end{array}$

system.

[4] TagMaster, NedTrain, and Bass: Gotcha system-Wheel flat detection and axle load measurement. http://www.tagmaster.com

[5] Strainstall: TrackAlert. http://strainstall.com/rail.html

[6] Schenck measuring process systems: Multirail: WheelScan. http:/www.schenck-process.de/catalogue/multirail.pdf

[7] Salient Systems Inc: (WILD) Wheel Impact Load Detector. http://www.salientsystems.com/prod_wild.html

[8] ØDS-CALTRONIC A/S: Wheel Monitoring System (WMS) detects impact defects and other tread irregularities. http://www.odegaard.dk/Caltronic/Default.htm

[9] Imagemap inc: wheelspec ${ }^{\circledR}$. http://www.imagemap.com/WheelSpec.html

[10] BHP institute of Railway Technology - Monash university: Wheel impact monitor (WIM). www.eng.monash.edu.au/railway

[11] Jodon and inc: Microwave Train Wheel Defects Measurement System. http://www.jodon.com/wheel.html 
[12] A. Bracciali and G. Cascini, A wheel flat detection device based on cepstrum analysis of rail acceleration measurements. World congress on Railway Reserarch WCRR97, Firenze, pp. 513-521, 1997

[13] A. Bracciali and G. Cascini, Detection of corrugation and wheelflats of railway wheels using energy and cepstrum analysis of rail acceleration, Journal of Rail and Rapid Transit, 211,pp. 109-116, 1997

[14] S. Lechowicz and C. Hunt, Monitoring and managing wheel condition and loading. International Symposium on Transportation Recorders, May 3-5 1999, 1999

[15] Qibo Feng et al, A dynamic and quantitative method for measuring wheel flats and abrasion of trains. 15th World Conference on NondestructiveTesting (WCNDT), Rome, 2000

[16] C.A. Papadopoulos, N.T. Niakas, and K. Diplari, Flatness identification in wheels of passing trains. 4th European Conference on Noise Control (Euronoise 2001), 2001

[17] A. Bracciali, G. Lionetti, and M. Pieralli, Effective Wheel Flats Detection Through A Simple Device. Proceedings of the 2002 Techrail Workshop, Paris, 2002

[18] D. Skarlatos, K. Karakasis, and A. Trochidis, Railway wheel fault diagnosis using a fuzzy-logic method, Applied Acoustics, 65,pp. 951-966, 2004

[19] TEKNIS ELECTRONICS: Wheel Condition Monitor (WCM). http://www.teknis.net

[20] B.P. Bogert, M.J.R. Healy, and J.W. Tukey, The quefrency analysis of time serie for echoes: Cepstrum, pseudo-autocovariance, cros-cepstrum and saphe cracking. Proceedings of the symposium on time series analysis, pp. 209-243, 1963

[21] A. Bracciai and F. Piccioli, Attenuation of rail vibration: Analysis of experimental data. Proceedings of the 5th World Congress on Railway Research, Koln, 2001

[22] D.J. Thompson, Experimental analysis of wavepropagation in railway tracks, Journal of sound and Vibration, 203(5),pp. 867-888, 1997 